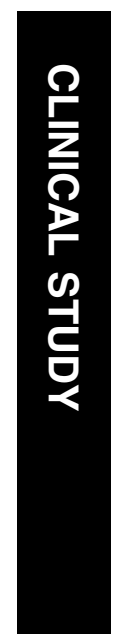

\section{Barriers to adherence with glaucoma medications: a qualitative research study}

should focus on investigating methods by which initial education about glaucoma and its management should best be delivered to patients.

Eye (2009) 23, 924-932; doi:10.1038/eye.2008.103; published online 25 April 2008

Keywords: adherence; glaucoma; qualitative research

Introduction

Poor adherence undoubtedly contributes to glaucoma remaining the third most common cause of blindness in Western populations. ${ }^{1-3}$ Adherence issues are complex, Tsai et $a l^{4}$ classifying as many as 71 adherence barriers into four categories - regimen, individual patient factors, medical provider issues, and situational factors.

Despite the recent call for further research relating to adherence with glaucoma therapy ${ }^{5}$ and growing acceptance/encouragement for the use of qualitative methods, ${ }^{6}$ there has been only one previous study performed from an in-depth qualitative perspective ${ }^{7}$ - somewhat surprising considering that assessment of adherence barriers relies primarily on patient's thoughts.

Taylor et $\mathrm{al}^{7}$ identified certain barriers to adherence for US patients, but the results were inevitably, culturally bound, and cultural factors influence adherence. ${ }^{2}$ Although many aspects relating to adherence may be similar in the United Kingdom and the United States, elements of cultural disparity and structural differences between health-care systems may affect health beliefs, attitudes, and hence, adherence. Although it was not the intention of the present study to examine explicit cultural 
differences between the United States and the United Kingdom, these differences highlight the value in conducting a UK study, similar to that of Taylor et al. ${ }^{7}$ Furthermore, Taylor et $a l^{7}$ highlighted the need for identification of motivational factors for adhering to glaucoma medication, an aim of the present study.

\section{Materials and methods}

Participants were recruited equally from two differing National Health Service (NHS) ophthalmic departments: the Norfolk and Norwich University Hospital (a teaching hospital serving urban and rural patients) and Cromer and District Hospital (serving rural patients). Participants either attended a focus group or had an interview. In total, 24 participants were recruited -7 to each focus group and 10 to interviews. The selected inclusion criteria (Table 1) ensured that participants had a significant clinical reason for adherence and were administering $\geqslant 2$ medications to increase the 'adherence effect' and had used drops for $\geqslant 1$ year to enable analysis of longer-term behaviour.

Two focus groups were conducted, one at each hospital. Participants attended the focus group at their

Table 1 Inclusion/exclusion criteria

\begin{tabular}{ll}
\hline Inclusion criteria & Exclusion criteria \\
\hline $\begin{array}{l}\text { Previous diagnosis of } \\
\text { moderate/severe POAG }\end{array}$ & $\begin{array}{l}\text { Inability to speak English } \\
\text { fluently (to eliminate potential } \\
\text { bias by interpretation of } \\
\text { information by a translator) }\end{array}$ \\
$\begin{array}{l}\text { Prescribed and advised to } \\
\text { administer } \geqslant 2 \text { topical }\end{array}$ & $\begin{array}{l}\text { Desire to withhold } \\
\text { demographic details }\end{array}$
\end{tabular}

anti-glaucoma medications

Visual acuity $\geqslant 6 / 12$

Clinically significant, serious, or severe medical or psychiatric condition

Previous consultations with $\geqslant 2$ ophthalmologists

Significant non-glaucomatous co-morbidity (eg macular degeneration)

Diagnosis of glaucoma

for $\geqslant 1$ year

Male or female $>$

18 years of age

Provision of a signed, ethics

committee approved, informed

consent

Abbreviation: POAG, primary open angle glaucoma.

aThe study definition of POAG included the presence of (1) a glaucomatous visual-field defect (Mean Defect worse than or equal to $-5.00 \mathrm{D}$ ) as detected by automated visual-field analysis (SITA-S 24/2, Humphrey Field Analyzer-II, Zeiss-Humphrey Instruments, Carl Zeiss Meditec Inc., Dublin, CA, USA), (2) documented previous elevated intraocular pressure ( $>22 \mathrm{~mm} \mathrm{Hg}$ ), and (3) a characteristic glaucomatous optic disc appearance. own hospital, held in a room away from the clinic for both comfort and to 'dilute' the effect of the clinic's environment. The focus groups were used to stimulate interaction between participants by asking open questions about experiences with therapy (Figure 1) so they could share/compare opinions and discuss any important areas not previously identified. The group meetings lasted for $2 \mathrm{~h}$ (longer than intended). However, concentration was well maintained, leading to an abundance of high-quality data. The information gathered from the focus groups ensured that all significant issues were incorporated into the interview guide, so that it reflected both theory and participant opinion (eg 'education' was added, appearing as an area of concern).

Following the focus groups, 10 one-to-one, homebased, semi-structured, in-depth interviews of 40-90 min were held, allowing collection of specific thoughts and feelings from individual participants. The interview guide (Figure 1) provided only a basis for discussion. All interviews were conducted (and taped) by one psychologist (JL), who worked in isolation from the clinic staff. Anonymity of involvement and interview dialogues from clinic staff was emphasized to allow 'free' discussions.

The written transcripts were sent to all participants for 'member checking' as described by Lincoln and Guba. Participants were invited to amend/remove any statements (within 10 days). Only a few minor alterations were received (all being added to the analysis), confirming data reliability. The final data consisted of interview audio tapes and transcripts (81 463 words), researcher notes, and member-checking correspondence. Data were annotated with patient initials ensuring anonymity.

The study had Suffolk Research Ethics Committee approval, followed all applicable institutional/ governmental regulations concerning ethical use of human volunteers and adhered to the tenets of the Declaration of Helsinki. All participants provided written consent.

\section{Analysis}

Data were analysed using 'Framework' analysis, ${ }^{9}$ a technique not requiring saturation of data, which is useful from both diagnostic (examining the reasons for, or causes of, what exists) and strategic objectives (identifying new theories, policies, plans or actions) that reflected study aims. The primary analysis, performed by the principal investigator (JL), was validated by a co-investigator (HC), as recommended by Armstrong. ${ }^{10}$ NVivo7 Software (QSR International Pty Ltd., Doncaster, 
1. Can you tell me a bit about yourself?

Hobbies/ Interests

2. How did you first come to learn that you had glaucoma?

\section{Medication}

3. Think back to the first time you used eye drops.

What were your first impressions?

\section{What has using eye drops been like for you?}

5. How do you feel about the eye drops you are currently taking?

How do you feel about the dosages?

What do you like most about the drops you use?

What do you like least about them and why?

What drawbacks / side effects, if any, are there to the eye drops?

\section{Attitudes and Behaviours}

\section{Memory \& Routine}

6. How much of an issue is remembering to take your eye drops?

Tell me about the last time you forgot to take your eye drops.

Why was that?

Can you tell me about anything that helps you remember to take your eye drops?

Why do you feel this helps you?

To what extent does this help you?

Can you tell me what, if anything, you would advise another glaucoma patient who was having problems remembering to take their eye drops?

7. Have you experienced any problems in your day to day life by taking your eye drops?

Can you explain why this has been a problem?

Can you think of any ways in which this problem could be reduced?

How do you feel when you take your eye drops regularly? How about when you do not?

Motivations

8. Would you say there is anything that motivates you to take your eye drops?

In what way does this motivate you?

How often does this motivate you?

Has this always motivated you? If not, what used to motivate you before this?

What could motivate you further?

\section{Practitioner}

14. To what extent do you think your doctor is serving your best interests with the eye drops he/she has chosen? Why do you feel that way?

15. Can you tell me about any personal involvement you have had in helping to choose your plan of care? What would you do differently if you could?

Education

16. Can you tell me about the level of advice you have been given in terms of teaching you about your glaucoma?

Who has given you the advice?

What help have you received?

Could anything more be done to help you understand?

17. Do you feel there is any other service the NHS could provide you with to help you take your drops more effectively?

\section{Coverage and Cost}

18. How do you feel about any payments you have had to make for eye drops?

Have you ever gone without your eye drops due to any financial costs?

Summary Questions

19. Summarise discussion....then ask; "How well does that capture what has been said here today?"

20. Of all the points we have discussed today, which one is most important to you?

21. Have I missed anything? Is there anything you came wanting to say that you didn't get a chance to say?

Figure 1 Interview guide. The interview schedule was loosely based on that used by Taylor $e t$ al with the addition of questions concerning lack of symptoms, motivations, and ideas to help future adherence. Questioning followed a narrative approach whereby patients initially discussed their pathway to diagnosis and glaucoma background followed by the delivery of key questions to determine attitudes/behaviours towards adherence and motivations for adherence. Finally, the patients were given the opportunity to suggest their ideas for improved adherence. 
Australia) was used to organize and reliability-check data.

\section{Results}

Participant demography is summarized in Figure 2.

Six main themes emerged from the transcripts, which explained obstacles to adherence. Details for each theme are provided below using quotations from the interviews (Table 2), each barrier being derived directly from participant experiences.

\section{Lack of education}

Few participants appeared fully satisfied with the level of glaucoma education that they had received. Both at diagnosis and follow-up, the provision of leaflets was the commonest method utilized, but this was felt by many to be inadequate, the desire being for more discussion about treatment. Other participants had received help on a request-only-basis and a few reported receiving no help.

Unsatisfactory hospital-led education was considered to be the direct result of the current NHS system, where doctors appeared too busy clinically to have time to provide adequate education. Furthermore, it appeared that doctor-patient communication issues such as the overuse of medical terminology or the use of different drop names often caused confusion. Many participants had self-educated using pharmacy leaflets, articles, pharmaceutical industry information or websites (quote 1). However, key knowledge about glaucoma still appeared lacking for two participants despite experience of the disease for $>1$ year (one participant reported being unaware whether or not glaucoma was fatal, having known someone who died shortly after trabeculectomy). Some participants also discussed how they felt common misconceptions about glaucoma within the community needed addressing.

In light of these issues, many participants expressed a desire for improved delivery of education. Participants emphasized the need for advice at diagnosis and during the first few consultations, both to relieve worry/shock and to provide correct information so that they might self-manage effectively from diagnosis (quote 2).

Participants discussed how education should concentrate on the consequences of poor adherence, drop application techniques, and advice about application

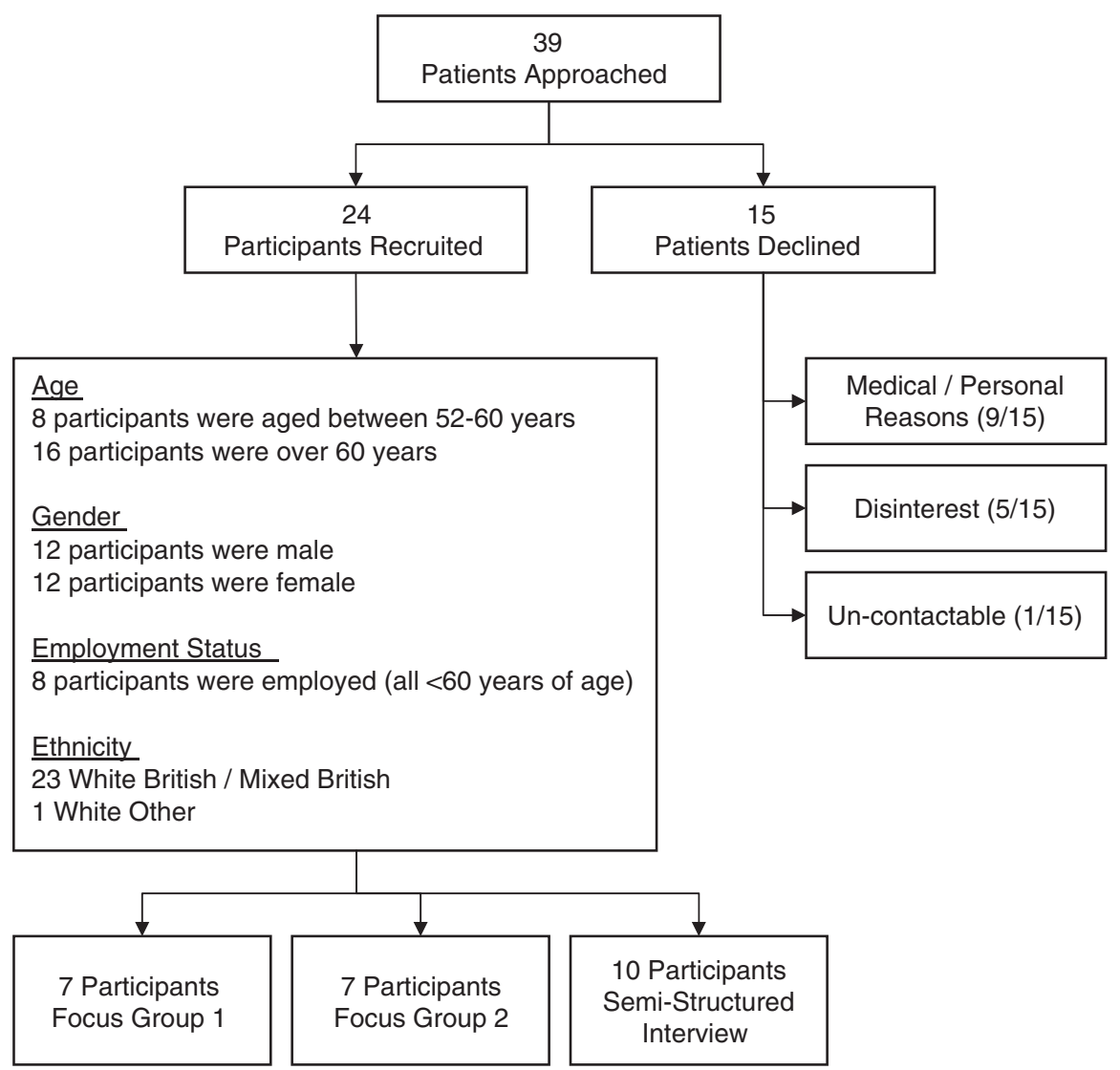

Figure 2 Recruitment demography. Recruitment demography shown as a flow chart. 
Table 2 Table of quotes

\begin{tabular}{|c|c|}
\hline $\begin{array}{l}\text { Quote } \\
\text { number }\end{array}$ & Quote \\
\hline 1 & I've got more information from the glaucoma association in their leaflets than I've had from the hospital. (KS) \\
\hline 2 & $\begin{array}{l}\text { When you are first diagnosed, it's an absolute blow isn't it?...you're stunned...from there on you need more information to } \\
\text { ease you into it what's going to happen or what has happened...not just give you some drops and say take them home and } \\
\text { do this three times a day. Especially when you're not quite sure how to do it. (SU) }\end{array}$ \\
\hline 3 & $\begin{array}{l}\text { Perhaps the first couple of times it might be an idea if someone else just approaches the patient and says' well are you } \\
\text { having any difficulty with the eye drops, having difficulty remembering to apply them, you know do you feel they are } \\
\text { working as they should do, are you possibly using too much, have you had this problem? It's just, because that would just I } \\
\text { would have thought just ease a lot of peoples problems that they have, just iron those problems out at that stage. (KB) }\end{array}$ \\
\hline 4 & $\begin{array}{l}\text { Well I'd like more. I mean I think you're treated like an idiot sometimes. I'd like it explained more. Diagrams drawn or } \\
\text { something like that. I'd like a more in depth explanation cos I don't totally understand it. (KS) }\end{array}$ \\
\hline 5 & $\begin{array}{l}\text { Yeah, it's just something you have to do. If you don't you'd end up with no sight probably ... so you just do what you have } \\
\text { to. (MW) }\end{array}$ \\
\hline 6 & $\begin{array}{l}\text { If people who haven't had any deterioration but their pressures are high, haven't lost any sight, they don't perhaps } \\
\text { appreciate the outcome if the miss them (their drops) as much. (SL) }\end{array}$ \\
\hline 7 & I love my babysitting. I think if there was a day when I couldn't do that, that would be awful. (AF) \\
\hline 8 & $\begin{array}{l}\text { I must admit last night I didn't so much as forget, I just couldn't be bothered to put them in (laughs) bloody things aren't } \\
\text { working anyway, the pressures up so I think 'why bother?, it's just a ritual I'm going through. But I've got no confidence } \\
\text { that they're doing anything to be honest. (KS) }\end{array}$ \\
\hline 9 & $\begin{array}{l}\text { Well, I must admit, openly here, I didn't have a clue what I was supposed to be doing. I'm sure in the first } 6 \text { months the first } \\
\text { probably } 2 \text { or } 3 \text { bottles were wasted 'cos they never went in where they were supposed to go. (SU) }\end{array}$ \\
\hline 10 & I feel even now, two years on whatever it is, there's sometimes too much going in. (FA) \\
\hline 11 & $\begin{array}{l}\text { For a young person it's not a problem but when you get to our age, we haven't got the pressure in our fingers to do it, you } \\
\text { know what I mean, it's like trying to open a bottle or something. And we have to have a special tool to open the top so; I } \\
\text { just haven't got the grip anymore. (CM) }\end{array}$ \\
\hline 12 & You were not physically shown how to do it. (BG) \\
\hline 13 & After the years I've just got used to it and I think that isn't gonna make that much difference jus \\
\hline 14 & It sends my blood sugar level up... because you know you've got to have them. You can't afford not to have them. (CM) \\
\hline 15 & $\begin{array}{l}\text { If you've got a daily rhythm of things you do at certain times it's easy. I think it's when you go and do other things that take } \\
\text { you away from the general routine, then you might forget it. (MW) }\end{array}$ \\
\hline 16 & $\begin{array}{l}\text { I always do them in the evening... Mornings would be the time that I would forget to do them... (It's then that) I've got a lot } \\
\text { on. Doing a lot. Rushing off to see somebody or something. (JG) }\end{array}$ \\
\hline 17 & $\begin{array}{l}\text { The biggest problem is when I was having them two-hourly, you had to really remember that. But now you've got to have } \\
\text { them at breakfast time and tea time and then supper time, that seems easy enough to remember. (MN) }\end{array}$ \\
\hline 18 & $\begin{array}{l}\text { I mean it's just the ones where you have to take them during the day if you go out. I mean you don't want to take eye drops } \\
\text { and put them in during the day... I don't mind mornings and nights but it is during the day that you think, 'Oh, I've got to } \\
\text { take them' or unless you miss them out, which sometimes I have done cos they do sting your eyes and make your eyes } \\
\text { water. (HP) }\end{array}$ \\
\hline 19 & We haven't got the pressure...I don't have any strength in my fingers. (CM) \\
\hline 20 & Old people don't like computers! (Laughs) Well not all of them. (EM) \\
\hline 21 & $\begin{array}{l}\text { I suppose I feel like I'm standing at the bottom of Everest looking up (isn't it). I mean here I am, at } 52 \text { and I suppose I think } \\
\text { well, perhaps I'll last until } 80 \text { or so, so } 30 \text { years of eyes drops... it does seem a long while to take eye drops for. (SL) }\end{array}$ \\
\hline 22 & $\begin{array}{l}\text { I was particularly concerned because I was young, that when I went to the clinic everyone else who was there was in their } \\
\text { sixties, seventies whatever and it's quite isolating to think you're the only one who's younger and all the related problems } \\
\text { such as driving, working, reading, the computer, wearing contact lenses, whatever, probably don't affect older people as } \\
\text { much. There just wasn't anybody else I could discuss this with at all. (AS) }\end{array}$ \\
\hline
\end{tabular}

schedules. Many proposed the introduction of a trained education assistant as a feasible way of providing advice, potentially improving adherence, and saving clinician time (quote 3). Participants also discussed education throughout follow-up as an area requiring expansion (quote 4).

\section{Lack of faith in drop efficacy}

Many participants had certain negative feelings about drops: they were 'paraphernalia' (EM) and the side effects could be unpleasant. However, it appeared that despite these issues, the majority continued to administer drops due to faith in drop efficacy preserving sight (quote 5), or because they already had symptomatic visual loss (quote 6). Understandably, sight preservation appeared to be the major motivation for adherence. All participants were emotional when imagining blindness and the anticipated effect of this for themselves/their families (quote 7).

However, it appeared that when efficacy or safety was doubted, motivation to persevere diminished. One 
participant described how he sometimes 'chose' not to administer drops (quote 8). Anticipated long-term side effects led another participant to undergo a supervised trial off therapy to prove that the drops were effective. Many participants desired more regular feedback about drop efficacy, so providing them with greater faith for adherence.

\section{Drop application issues}

Many participants recalled problems with drop administration soon after diagnosis and several discussed current problems. Problems appeared most poignant in the initial stages, with many participants recalling that uncertainty about application methods and difficulty in physically administrating drops reduced confidence and application accuracy (quote 9).

Later in care, participant opinion over-drop application was split; for some drop administration became simple and 'second-nature' (TE), while for others there were continued concerns with respect to correct use of medication. Operational and technique issues provided the main areas of concern with uncertainties often remaining chronically (quote 10). For example, some participants reported finding it awkward to apply drops correctly, sometimes flooding the eye or face. Additional physical problems with grip, balance, and precision were highlighted by some older participants (quote 11).

Both initial and chronic application issues were considered to have arisen from poor early drop application education. Few participants had received any guidance with respect to effective administration (quote 12). As a result, participants had had to rely on instructions provided with their medication, often considered difficult to read, or confusing as it conflicted with information obtained from other sources (eg internet, pharmacists, and other patients). The need for drop administration advice at diagnosis was discussed far more than any other topic, with many participants proposing to ask their practitioner to check their current technique.

\section{Forgetting drops}

All but two of the participants admitted to forgetting drops at some stage. For the majority, the frequency of forgetting appeared to be split between those who occasionally forgot and those who rarely forgot. There were marked attitude differences between the feelings of those who frequently, and those who rarely, forgot. While the majority of frequent/occasional forgetters were in agreement that if they forgot they would not particularly worry (quote 13), most of the participants who never/ rarely forgot appeared concerned (quote 14). In addition, focus-group participants appeared to admit to forgetting drops more freely after others had done so, many discussing this in a light-hearted manner. In contrast, interview participants tended to stick with their original comments.

For all participants, forgetting drops appeared to be explained by factors influencing routine. All reported a reliance on daily events such as bedtime or meals to act as cues. For some participants clock-time was their only cue. Events (eg holidays) that altered normal routine were the most commonly reported reasons for poor adherence (quote 15). In addition, particular doses, those prescribed to be taken at busiest times of day or doses that failed to fall naturally into a daily regimen, were more 'at risk' of being forgotten (quote 16). A complex dosing regimen with a greater quantity of doses, for example with the addition of post-operative drops, had an adverse effect (quote 17).

Several other cues and memory aids were described, including the assistance of a spouse, a chart or alarm. However, these methods all appeared to have been used in conjunction with habits and/or clock-time.

\section{Practical problems}

Participants discussed several practical issues acting as barriers to adherence. Some had been untreated for short periods having run-out of drops, failing to re-order drops or having experienced problems obtaining new drops before current bottle expiry. Others who had been prescribed drops to be taken during the day, rather than early-morning or late-evening, admitted adherence difficulties. Problems included difficulties in finding a convenient location (often due to embarrassment), having inadequate time to apply the drops, keeping drops cool, eyes watering/stinging while trying to perform daily activities, and remembering drops when busy (quote 18).

Several participants complained about medication packaging, side effects, and prescription costs (for employed participants, $<60$ years). However, although these issues were described as frustrating and potentially off-putting to participants, none were explicitly associated with non-adherence.

\section{Age and individual differences}

Both individual and age differences were applicable to all themes. By classifying participants as 'older' ( $\geqslant 60$ years) or 'younger' ( $<60$ years) and comparing attitudes, it became evident that 'older' patients more often required additional assistance, with many anticipating, or indeed experiencing, greater difficulty with physically applying 
and remembering drops (quote 19). Conditions associated with older age were also anticipated to reduce adherence (eg tremor, arthritis). Some older participants discussed difficulty in accessing external educational resources, particularly websites (quote 20). In contrast, many of the 'younger' participants felt cheated or depressed, considering glaucoma to be an 'older person's disease'. Many discussed concern about life-long treatment (quote 21) and a feeling of isolation in clinics when surrounded by older patients. The desire for a 'young-only' glaucoma group where young patients could exchange specific age-related advice was widely suggested (quote 22). Furthermore, 'younger' participants felt they were treated differently by staff, with some feeling hospital staff were overly helpful, and others feeling they were less supportive. Age-related issues identified by younger participants emerged during interviews and not in the focus groups. As a taboo subject, age may not have been discussed in focus groups to avoid offence.

Individual differences were evident across all themes. Opinions differed according to educational needs, degree of initial, and ongoing application problems, tendency and feelings towards forgetting drops, and adherence cues (including routine). Further practical problems and age-related differences also varied with individual lifestyle. Participants widely discussed the idea of 'tailoring' management with respect to age and individual differences, both to maximize the use of clinical time and maximize adherence.

\section{Discussion}

The present study was the first UK-based, qualitative investigation of adherence with anti-glaucomatous therapy. Multiple obstacles to adherence were identified through an extensive 'Framework' analysis of participants' thoughts/feelings, collected from both focus-groups and individual interviews. Lack of initial education about application techniques, consequences of non-adherence, and drop-scheduling issues were regarded as key factors. Patients appeared to need: sufficient initial information, long-term education (with feedback), and the establishment of sufficient medication-faith to enable self-management. Although the fundamental motivation to adhere was sightpreservation, two 'exceptions' demonstrated that adherence can lapse when drop efficacy is doubted. Difficulties with drop application, forgetting drops, and other practical issues including scheduling, as well as problems with drop supply, were other more common barriers to adherence. Furthermore, individual and age-related differences were identified for all obstacles; hence, the suggested 'tailoring' of adherence advice.
Many findings were similar to those of the Taylor et $a l^{7}$ US-based study. Since the majority of participants in both studies admitted non-adherence, with forgetfulness and application issues identified as two major problems, the importance of addressing these issues should be emphasized. Our study answered Taylor's question: 'What factors motivate patients to be adherent and what could motivate them further $?^{\prime 7}$ Fear of blindness appeared to be the universal motivation for adherence, explaining why lack of faith in drop efficacy had a negative influence. The results highlighted the importance of providing feedback about medication efficacy and how a move away from an often inappropriate 'fear of blindness' driver to adherence would be ideal.

Adherence levels appeared reasonable for our group of patients (the majority forgetting drops infrequently), drop administration problems being resolved soon after diagnosis, and a large number of additional practical problems being overcome with time. Adherence in our study seemed to be linked to a common fear of visual loss, inducing self-motivated perseverance, identification of memory cues, and solutions to various practical/ logistical problems. The fact that all participants administered drops using 'routine' was an example of such motivation.

However, since many participants experienced periods of poor adherence and recalled problems as newly diagnosed patients, changes in clinical practice appear necessary. Improving clinical practice is particularly important given adherence levels are currently underestimated by clinicians and patients. ${ }^{11-13}$ Our findings may be useful to both clinicians and patients in promoting adherence, identifying individual causes of non-adherence as early as possible after diagnosis and stimulating appropriate discussion to find potential solutions. Emphasis should be placed on identification of the stage(s) where adherence is breaking down for an individual patient so that tailored additional assistance and advice can be provided. Berger ${ }^{14}$ suggested the use of motivational interviewing to improve adherence; a move towards a psychosocial model of care (rather than the traditional biomedical approach). Future development of a health behaviour model specific to glaucoma patients should therefore combine both a promotional tool and treatment strategy aid for long-term management.

In addition, our results, like Taylor et $\mathrm{al}^{7}$ suggested that the introduction of an education assistant, working in glaucoma clinics would be a useful resource having the potential to combat obstacles to adherence. Further to Taylor et al, ${ }^{7}$ our results suggested that the emphasis of this assistance should be at diagnosis and should be tailored to the individual. Overall adherence levels could 
be improved by better initial education at diagnosis, together with long-term feedback at all stages. Understanding the consequences of non-adherence would appear to have the potential to establish motivation from the outset. Participants could be taught effective application techniques, inducing confidence. Memory aids/cues and the importance of routine could be discussed and administration schedules devised so that effective measures to ensure adherence are in place at commencement of treatment. Potential practical barriers would be addressed and overcome. Furthermore, by 'tailoring' education to the individual according to age and lifestyle, specific adherence issues would be targeted and potentially overcome in an appropriate and patient-centred manner.

Future research should focus on assessing the value of health behaviour models in improving glaucoma medication adherence and investigating methods by which initial education, drop application techniques, and advice on memory aids/cues should best be delivered to patients with glaucoma.

\section{Methodological critique}

From the rich amount of data acquired and the creation of six themes directly reflecting participant opinion, 'Framework' analysis and qualitative methodology successfully unearthed participant opinion. Use of both focus groups and interviews allowed the study to benefit from the dynamics attributed to both group discussion and individual interviews. Participants in the focus groups appeared encouraged by one another to admit to non-adherence, and yet the privacy of individual interviews, enabled discussion about age-related issues. In addition, the agreement between focus groups and interviews in terms of content and opinion added to study reliability. The acknowledgement and use of negative cases and accumulative techniques ${ }^{15}$ throughout the analysis increased validity and revealed the importance of diversity of opinion in the glaucoma patient population, which may have been overlooked using quantitative methods.

Qualitative research in particular can be criticized for being generalizable to only the population from which the participants were selected. The relationship between adherence and socio-demographic factors such as age, gender, race, and intelligence/education is complex, consequently random selection of participants was employed rather than selecting a sample in an attempt to represent the whole UK population. Unfortunately, due to low ethnic diversity and strict inclusion criterion (resulting in exclusion for clinical reasons of the few screened ethnic minority patients), all except one of the participants were white British (with one white-other).
The authors acknowledge that their findings may not apply to all the UK residents since ethnicity affects adherence $^{2}$ and would encourage further studies for different ethnic groups. However, apart from ethnicity, it was considered that the sample selected, provided a reasonable representation of the UK population with a wide age range, equal gender split, and variety of occupations that indicated different levels of education. Participants provided a broad representation of patient opinion and practical issues, relevant to the majority of the UK glaucoma patients under NHS care.

\section{Reflexive analysis}

Willig ${ }^{15}$ promoted the importance of both personal and epistemological reflexive analysis in qualitative research. On reflection 'Framework' analysis was considered appropriate in gaining an understanding of participants' experiences. Although it was accepted that the principal investigator (JL) was important in theme creation, the theories identified were grounded in participants' discourse, were co-investigator verified, and as such were considered a good indication of real thoughts / perceptions. Since participant diversity and novel experiences were revealed and not anticipated, concerns regarding such bias were minimized.

Studies of adherence may be intrinsically biased though the selection of patients who attend follow-up appointments; non-adherent patients who suffer the key problems of adherence and ongoing care being more likely to drop-out of follow-up and be missing from random selection. While the authors were unable to account for those patients who had dropped out of care, by noting the number of patients approached and explanatory reasons for non-participation (Figure 2), it can be seen that the majority of participants approached agreed to take part, this including some self-confessed poorly adherent participants who contributed to the findings. However, since the sample was theoretically the more adherent patients (those attending follow-up and agreeing to participate), the magnitude of adherence concern is likely to be under-reported - thus, caution is warranted in extrapolating these results.

Participants may have felt uneasy about admitting non-adherence. Although anonymity from clinical staff was guaranteed and the principal investigator was a non-medically trained health professional, nonadherence may have been under-reported. However, in view of the large amount of data collected, a good rapport throughout all interviews and the admission by many of non-adherence, our findings were considered to be valid. The focus group and interview locations were away from the clinic environment, facilitating the process and 'diluting' clinician presence. Finally, many 
participants reported greater awareness of adherence problems and approved of the opportunity to discuss their concerns demonstrating how the participants themselves benefited from the study.

\section{Conclusion}

Multiple barriers to adherence with anti-glaucomatous therapy were classified, which may aid their identification in future clinical practice. Findings suggest that specific obstacles to adherence should be identified soon after diagnosis and patients educated in how to tackle them. Use of health behaviour models and introduction of a glaucoma education assistant may facilitate the development of a tailored approach, accounting for individual and age-related differences in preventing poor adherence. Future research should focus on investigating methods by which initial education about glaucoma and its management, should best be delivered to patients.

\section{Acknowledgements}

The study was funded by the Norwich Glaucoma Research Fund. We thank Tom Eke, Consultant Ophthalmologist at the Cromer and District Hospital, for identifying suitable participants, staff at both the Norfolk and Norwich University Hospital and the Cromer and District Hospital for allowing recruitment from their clinics and, above all, the participants for their time and input.

\section{References}

1 Olthoff CMG, Schouten JSAG, Bart W, Webbers CAB. Noncompliance with ocular hypertensive treatment in patients with glaucoma or ocular hypertension, an evidence based review. Ophthalmology 2005; 112: 953-960.

2 Ethical Strategies Ltd.. The costs of blindness. An analysis of the costs of visual impairment and blindness in the United
Kingdom. [serial online] 2003 [cited 2006 Sept 30]; [1-65]. Available from URL: http://www.healthyeyes.org.uk/ fileadmin/healthy-eyes/downloads/costs-ofblindness.doc.

3 Mitchell P, Smith W, Attebo K, Healy PT. Prevalence of open angle glaucoma in Australia: the blue mountains study. Ophthalmology 1996; 103: 1661-1669.

4 Tsai JC, McClure CA, Ramos SE, Schlundt DG, Pichert JW. Compliance barriers in glaucoma: a systematic classification. J Glaucoma 2003; 12: 393-398.

5 Quigley H, Goldberg I, Kitazawa Y, Halprin R, Hitchings R, Gross R et al. Cooperation with medical therapy, report from the global AIGS committee on cooperation with therapy. In: Greve EL (ed). International Glaucoma Review, Supplement 2006, vol. 7. Kugler Publications: The Netherlands, 2006, pp 354-359.

6 Green J, Britten N. Qualitative research and evidence based medicine. BMJ 1998; 316: 1230-1232.

7 Taylor SA, Galbraith SM, Mills RP. Causes of noncompliance with drug regimens in glaucoma patients: a qualitative study. J Ocul Pharmacol Ther 2002; 18: 401-409.

8 Lincoln YS, Guba EG. Naturalistic Inquiry. Sage: Beverly Hills, 1985, pp 314-316.

9 Ritchie J, Spencer L. Qualitative data analysis for applied policy research.In: Bryman A, Burgess R (eds). Analysing Qualitative Data. Routledge: London, 1994, pp 173-194.

10 Armstrong D, Gosling A, Weinman J, Marteau T. The place of inter-rater reliability in qualitative research: an empirical study. Sociology 1997; 31: 597-606.

11 Zimmerman T, Zalta A. Facilitating patient compliance in glaucoma therapy. Surv Ophthalmol 1993; 28: 252-257.

12 Roth H, Caron H. Accuracy of doctors' estimates and patients' statements on adherence to a drug regime. Clin Pharmacol Ther 1978; 23: 361-370.

13 Kass M, Gordon M, Meltzer D. Can ophthalmologists correctly identify patients defaulting from pilocarpine therapy? Am J Ophthalmol 1986; 101: 524-530.

14 Berger BA. Improving treatment adherence (medication compliance) a motivational interviewing approach. [serial online] 2007 [cited 2007 August 10]; [1-33]. Available from URL: https://webapp.walgreens.com/cePharmacy/ programsHTML/compliance.html.

15 Willig C. Introducing Qualitative Research in Psychology: Adventures in Theory and Method. Open University Press: Buckingham, 2001, pp 16-19. 